Highly Energetic Physical Processes and

Mechanisms for Emission from Astrophysical Plasmas

IAU Symposium, Vol. 195, 2000

P. C. H. Martens, S. Tsuruta, and M. A. Weber, eds.

\title{
NLTE Model Atmospheres for Extremely Hot Compact Stars
}

\author{
T. Rauch, J. L. Deetjen, S. Dreizler, and K. Werner \\ Institut für Astronomie und Astrophysik, Univ. Tübingen, Germany
}

\begin{abstract}
Present observational techniques provide stellar spectra with high resolution at a high signal-to-noise ratio over the complete wavelength range-from the far infrared to X-rays.

The effects of Non- "Local Thermal Equilibrium" (NLTE) are particularly important for hot stars, hence the use of reliable NLTE stellar model atmosphere fluxes is required for an adequate spectral analysis.

State-of-the-art NLTE model atmospheres include metal-line blanketing of millions of lines of all elements from hydrogen up to the irongroup elements, and thus permit precise analyses of extremely hot compact stars, e.g., central stars of planetary nebulae, PG 1159 stars, white dwarfs, and neutron stars. Their careful spectroscopic study is of great interest in several branches of modern astrophysics, e.g., stellar and galactic evolution, and interstellar matter.
\end{abstract}

\section{Introduction}

During their evolution, the more massive post-AGB (post-"Asymptotic Giant Branch") stars can reach extremely high effective temperatures: up to about $700 \mathrm{kK}$ are predicted by Paczynski (1970) for a star with a remnant mass of $1.2 \mathrm{M}_{\odot}$. Due to their flux maximum in the EUV/X-ray wavelength range, and depending on their photospheric composition and interstellar absorption, some post-AGB stars have been detected as supersoft X-ray sources, e.g., all PG 1159 stars with $T_{\text {eff }}>140 \mathrm{kK}$ have been detected by ROSAT. Realistic modeling of the emergent fluxes of these stars is a prerequisite for a reliable spectral analysis.

\section{NLTE Model Atmospheres}

The model atmospheres (plane-parallel, hydrostatic and radiative equilibrium) are calculated using the code PRO2 (Werner 1986; Werner 1988). All elements from hydrogen to the iron group are considered (Rauch 1997; Dreizler \& Werner 1993; Deetjen et al. 1999).

The high-energy model atmosphere fluxes strongly depend on the metal-line blanketing. The impact of $\mathrm{H}-\mathrm{Ca}$ is shown in detail by Rauch (1997). A grid of model atmosphere fluxes $\left(T_{\text {eff }}=50-1000 \mathrm{kK}, \log g=5-9(\mathrm{cgs}), \mathrm{H}-\mathrm{Ca}\right.$, solar and halo abundances) is available on the WWW (http://astro.uni-tuebingen.de/ rauch/flux.html). In Figure 1, we show the additional impact of the iron group. 


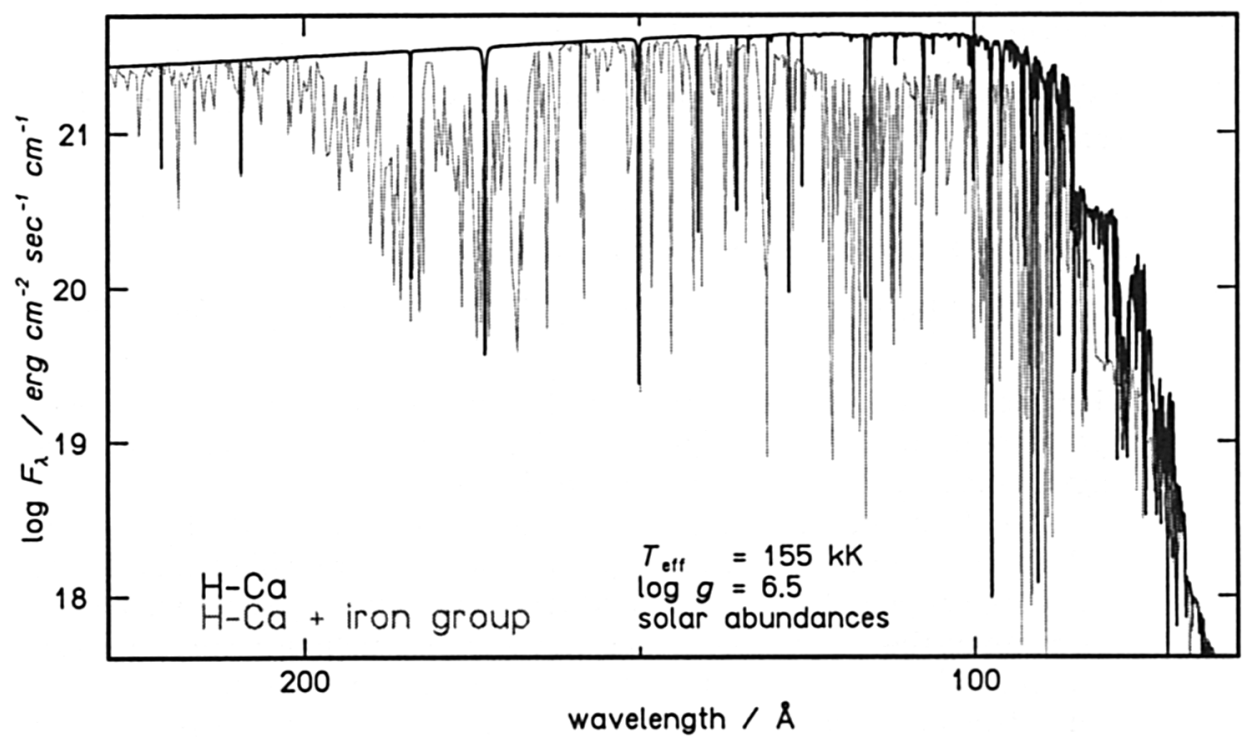

Figure 1. Comparison of two NLTE model atmosphere fluxes without and with consideration of iron-group elements. The additional iron-group line blanketing has a strong influence on the flux level at high energies close to the maximum flux level.

\section{Conclusions}

Emergent fluxes calculated from NLTE model atmospheres which include irongroup line blanketing show a drastic decrease of the flux level at high energies.

For a reliable analysis of UV/EUV and X-ray spectra of extremely hot stars, or the calculation of ionizing spectra from these (e.g., used as input for photoionization models), the consideration of all elements from hydrogen up to the iron group is recommended. A detailed consideration of metal-line blanketing with all available lines has an important influence on the spectrum.

Acknowledgments. This research is supported by the DLR under grant 50 OR 97055.

\section{References}

Deetjen, J. L., Dreizler, S., Rauch, T., \& Werner, K. 1999, A\&A, 348, 940

Dreizler, S., \& Werner, K. 1993, A\&A, 278, 199

Paczynski, B. 1970, Acta Astron., 20, 47

Rauch, T. 1997, A\&A, 320, 237

Werner, K. 1986 A\&A, 161, 177

Werner, K. 1988, A\&A, 204, 159 